\title{
Association of Fluid Overload with Mortality in Critically-ill Mechanically Ventilated Children
}

\author{
Sukla Samaddar, Jhuma Sankar, Sushil Kumar Kabra ANd Rakesh LodHa \\ From Department of Pediatrics, All India Institute of Medical Sciences, New Delhi, India. \\ Correspondence to: Dr Rakesh Lodha, Professor, Department of Pediatrics, All India Institute of Medical Sciences, New Delhi, \\ India.rlodha1661@gmail.com \\ Received: May 08, 2017; Initial review: June 21, 2017; Accepted: July 31, 2018.
}

Objectives: To study the association of fluid overload with mortality and morbidity in critically-ill mechanically ventilated children.

Design: Prospective observational study.

Setting: Pediatric Intensive Care Unit (PICU) of a tertiary care hospital, New Delhi, India.

Participants: 118 children (age $1 \mathrm{mo}-15 \mathrm{y}$ ) requiring mechanical ventilation.

Outcome measures: Primary: Association of fluid overload with mortality. Secondary: Association of fluid overload with oxygenation, organ dysfunction, duration of mechanical ventilation and PICU stay.

Results: Cumulative fluid overload of $\geq 15 \%$ was observed in 74 $(62.7 \%)$ children. About $50 \%$ of these children reached cumulative fluid overload of $\geq 15 \%$ within the first 5 days of PICU stay. The mortality was $40.5 \%$ in those with $\geq 15 \%$ cumulative fluid compared to $34 \%$ in the rest [OR $(95 \% \mathrm{Cl}): 1.02(0.97,1.07)]$. On multivariate analysis, after adjusting for confounders, cumulative fluid overload $\geq 15 \%$ was associated with higher maximum PELOD (pediatric logistic organ dysfunction) score (Median: 21 vs. $12 ; P=0.03$ ), longer median duration of mechanical ventilation (10 vs. $4 \mathrm{~d} ; P<0.0001)$ and PICU stay ( 13.5 vs. $6 \mathrm{~d} ; P$ $<0.0001$ ). There was no significant association of fluid overload with oxygenation index $(P=0.32)$.

Conclusion: There is no association of fluid overload with mortality. However, it is associated with poor organ function, longer duration of mechanical ventilation and PICU stay in critically-ill, mechanically ventilated children.

Keywords: Intensive Care, Mechanical Ventilation, Outcome.
$\mathrm{F}$ luid balance is one of the most challenging aspects of critically-ill children requiring pediatric intensive care. While early and aggressive fluid resuscitation is lifesaving and widely practiced, excess fluid may be detrimental. Mechanically ventilated children may be more prone to fluid overload as they have lesser evaporative losses due to warmed, humidified gases and thermocontrolled environment [1]. Presence of non-osmotic stimuli, in addition to osmotic stimuli, for anti-diuretic hormone $(\mathrm{ADH})$ release may contribute to fluid retention $[2,3]$. In addition, overestimation of fluid requirement occurs if traditional methods designed primarily for healthy children are used [4].

The Fluids and Catheters Treatment Trial (FACTT) in adults concluded that, patients managed with fluid restriction had lesser duration of intensive care unit stay and mechanical ventilation than patients who received liberal fluids [5]. The observations from few retrospective studies in children suggest adverse effects of positive fluid balance on respiratory morbidity and mortality [6]. We conducted this study to evaluate the association of fluid overload with survival and morbidities in critically-ill mechanically ventilated children in a PICU setting. Our primary objective was to study the association of fluid overload (both at 48 hours and total duration of PICU stay) and mortality. Secondary objectives were to study the association of fluid overload with peak oxygenation index (OI), organ dysfunction using Pediatric Logistic Organ Dysfunction or PELOD score [7], duration of mechanical ventilation and length of PICU stay.

Accompanying Editorial: Pages 945-46.

\section{METHODS}

This prospective observational study was conducted in an eight-bedded PICU of All India Institute of Medical Sciences, New Delhi from July 2013 to March 2015. Children aged $>1$ month to $\leq 15$ years requiring mechanical ventilation for at least 24 hours were enrolled and followed-up for a maximum of 14 days and for final outcome during PICU stay. Children with congenital heart disease, acute or chronic renal failure, renal tubular 
defects, post renal transplant, those receiving peritoneal or hemodialysis, and those with advanced malignancies were excluded. Children who were mechanically ventilated $\geq 24$ hours prior to admission in PICU were also excluded due to uncertainty of previous course and fluid received. Only the first admission was considered for every patient, and readmissions were excluded. The study was approved by the Institutional Ethics Committee and written informed consent was obtained from either of the parents or legal guardian.

In our unit, twice daily documentation of ventilatory parameters and arterial blood gas values of patients is performed. Detailed documentation of patient's fluid input and output is a part of the daily nursing schedule. All enrolled children after initial fluid resuscitation (if required), received maintenance fluid therapy using isotonic fluids (dextrose normal saline) in most cases. The Holliday Segar formula [8] was used for initial calculation which was adjusted depending on the disease condition (children with CNS/hepatic diseases received approximately two-thirds of the calculated volume if hemodynamically stable), the input-output balance, need for correction for excessive or reduced insensible losses, and the further hemodynamic status of the child.

Fluid overload percent $(\mathrm{FO} \%)$ was defined as = [Total fluid input in 24 hours $(\mathrm{mL})$ - total fluid output in 24 hours $(\mathrm{mL}) /$ weight at admission $(\mathrm{g})] \times 100$. Cumulative fluid balance on any particular day was defined as the sum total of daily fluid balances till that particular day (minimum of 24 hours to maximum of 2 weeks). Significant fluid overload was defined as a cumulative fluid balance of $\geq 15 \%$ attained at any time point during the 14 day study period and peak fluid overload was defined as the maximum percentage cumulative fluid overload on any day during the study period. Baseline demographic characteristics including weight, height, body mass index (BMI) and percentage predictive mortality at admission using Pediatric Index of Mortality-2 (PIM2) score [9] were recorded. At admission to PICU, infants were weighed using an infant weighing scale, while older children were weighed using a weighing chair scale. We were able to weigh all the intubated children taking precautions to avoid extubation. Subsequently, daily input was calculated as the sum of the total parenteral [intravenous (IV) fluids, medications, boluses, fluid replacements and blood products] and enteral fluids (oral, nasogastric or orogastric) received. Fluid output was calculated as sum of daily urine output and other body fluid output (nasogastric aspirate/ drain, drain from intercostals, or other surgical drains); these were recorded using the nursing charts on a daily basis. Insensible losses and fecal fluid losses were not included. From these data, daily fluid balance was calculated and cumulative figures generated for the total duration of mechanical ventilation.

If any child had overt clinical features of fluid overload like pulmonary edema or increase in liver size, appropriate measures were taken depending on the perfusion status of the child. Example, in a normo- or hypertensive child with these clinical features, diuretics like furosemide was used and fluid infusion was restricted. On the other hand, if the child was in shock and developed these signs (may occur in children with septic shock and myocardial dysfunction), the primary goal was to maintain the perfusion, using inotropes and modifying the ventilator settings to ensure adequate oxygenation as well. On the other hand, there are no well-accepted management guidelines for children who have a positive fluid balance in absence of overt signs of fluid overload.

Daily documentation of ventilator parameters [respiratory rate, inspiratory time (Ti), peak inspiratory pressure (PIP), positive end expiratory pressure (PEEP), $\mathrm{FiO}_{2}$, mean airway pressure] was done. Ventilatory settings were standardized for all children as per PICU protocol at our institute; these were influenced by the lung conditions and modified based on clinical condition and arterial blood gas reports. Arterial blood gas analysis was performed using ABL Flex (Radiometer Copenhagen, Denmark) and required parameters $\left(\mathrm{pH}, \mathrm{PCO}_{2}\right.$, $\mathrm{PO}_{2}$ ) were documented. Also parameters required for determining PELOD score were recorded daily for an objective scoring of organ dysfunction .

Statistical analysis was performed using Stata 11.2 (Stata Corp, College Station, TX, USA). The relationship between the fluid overload and the outcome variables was examined using bivariate (chi-square tests for categorical data and $t$-tests for continuous variables) and multivariate analyses (logistic regression) for mortality, controlling for confounders such as age, gender, nutritional status, and severity of illness at admission (PIM2 score).

\section{RESUlTS}

During the study period, 278 children required mechanical ventilation, out of whom 118 (58.5\% boys) were enrolled (Fig. 1). The median (IQR) age of the study participants was $14.5(4,72)$ months. Table I show the baseline characteristics. The most common indications for mechanical ventilation in patient management were septic shock (49.1\%), impending respiratory failure secondary to pulmonary causes $(24.6 \%)$ and $\mathrm{CNS}$ causes (11.1\%) followed by hepatic encephalopathy (3.4\%). At admission, children had a mean (SD) predicted mortality using PIM2 score of 15.1 (18.7). 


\begin{tabular}{|c|c|c|c|}
\hline Total admis & & Excluded & 160 \\
\hline 533 & & Mechanical ventilation $<24 \mathrm{~h}$ & 41 \\
\hline$\downarrow$ & & Inadequate data & 36 \\
\hline Total number of & & Age $<1 \mathrm{mo}$ & 25 \\
\hline mechanically & & Acute kidney injury & 21 \\
\hline ventilated & $\rightarrow$ & Congenital heart disease & 19 \\
\hline children & & Readmissions & 10 \\
\hline & & Chronic kidney disease & 5 \\
\hline$\downarrow$ & & Renal tubular acidosis & 2 \\
\hline $\begin{array}{c}\text { Enrolled } \\
118\end{array}$ & & $\begin{array}{l}\text { Mechanical ventilation } \geq 24 \mathrm{~h} \\
\text { outside PICU }\end{array}$ & 1 \\
\hline
\end{tabular}

FIG. 1 Flow of participants in the study.

Cumulative fluid overload of $\geq 15 \%$ was observed in $74(62.7 \%)$ children. Around $50 \%$ of children reached cumulative fluid balance of $\geq 15 \%$ within the first 5 days of PICU stay (Web Fig. 1). The median cumulative fluid overload percentage (IQR) was 19.6\% $(10.5 \%, 30.8 \%)$. The median (IQR) cumulative fluid overload at 48 hours was $7.9 \%(3.1 \%, 11.8 \%)$.

Of the enrolled children $38.1 \%$ (45/118) died. Among 74 children who had $\geq 15 \%$ cumulative fluid overload, mortality rate was $40.5 \%(30 / 74)$ as compared to $34 \%$ $(15 / 44)$ in those with $<15 \%$ cumulative fluid overload $(P=0.49)$ (Table II). The median (IQR) peak fluid overload in survivors was $10.2 \%(3.3 \%, 17.4 \%)$ as compared to $24.6 \%(13.6 \%, 35.5 \%)$ in non-survivors $(P=0.11)$. This was not significant on multivariate analysis $(P=0.50)$ (Web Table I).

At 48 hours, the median (IQR) peak fluid overload in survivors was $6.6 \%(3.1 \%, 10.3 \%)$ compared to $9 \%$ $(6.1 \%, 12.7 \%)$ in non-survivors $(P=0.04)$. However, on multivariate logistic regression analysis, there was no such association after adjusting for confounding factors $(P=0.12)$ (Web Table I). Only the PIM2 score at admission, PELOD score and diagnosis of sepsis showed significant association with mortality (Web Table I).

The maximum OI values were similar in children with
TABLE I Baseline Characteristics of Study ChILdRen $(N=118)$

\begin{tabular}{ll}
\hline Variables & Values $(n=118)$ \\
\hline Age (mo), median (IQR) & $14.5(4,72)$ \\
Male gender, $n(\%)$ & $69(58.5)$ \\
Weight Z score, median (IQR) & $-2.65(-4.85,-1.42)$ \\
Height Z score, median (IQR) & $-1.32(-3.87,-0.07)$ \\
BMI Z score, median (IQR) & $-2.78(-4.66,-1.28)$ \\
PIM2 score, mean (SD) & $15.08(18.65)$ \\
Admission diagnosis, $n(\%)$ & \\
$\quad$ Sepsis & $58(49.2)$ \\
$\quad$ Pulmonary & $29(24.6)$ \\
CNS & $13(11.0)$ \\
$\quad$ Hepatic & $4(3.4)$ \\
$\quad$ Non pulmonary infections & $4(3.4)$ \\
$\quad$ without sepsis & \\
Miscellaneous & $10(8.5)$ \\
\hline
\end{tabular}

$\geq 15 \%$ cumulative fluid overload [median (IQR): 10.3 $(5.4,18.0)]$ as compared to those with $<15 \%$ cumulative fluid overload [median (IQR): 11.1 (5.9, 21.4)] $(P=0.38)$.

Higher organ dysfunction as calculated using PELOD score was seen in those with $\geq 15 \%$ cumulative fluid overload [median (IQR): $21(11,31)$ ] as compared to children with $<15 \%$ cumulative fluid overload [median (IQR): $12(3.25,21.75)](P=0.03)$. On multivariate analysis, the association between fluid overload and PELOD score remained significant $(P=0.02)$ (Web Table II). Cumulative fluid overload at 48 showed no association with organ dysfunction $(P=0.31)$.

The duration of mechanical ventilation was significantly higher among children with $\geq 15 \%$ cumulative fluid overload [median (IQR): $10(6,16.2)$ days] compared to that in children with $<15 \%$ cumulative fluid overload [median (IQR): $4 \quad(2.25,8)$ days $(P<0.001)]$. On multivariate analysis, association remained significant $(P<0.001)$ after adjusting for confounding factors (Web Table II). Cumulative fluid

TABLE II Fluid Overload and Mortality in Mechanically Ventilated Children ( $N=118)$

\begin{tabular}{llll}
\hline Fluid overload & Survivors $(n=73)$ & Non-survivors $(n=45)$ & $P$ value \\
\hline $\begin{array}{l}\text { Cumulative maximum percentage fluid overload } \\
\quad \geq 15 \%, n(\%)\end{array}$ & $44(60)$ & $30(67)$ & 0.48 \\
$\quad<15 \%, n(\%)$ & $29(40)$ & $15(33)$ & $24.6(13.6,35.5)$ \\
Peak cumulative fluid overload \%; median (IQR) & $10.2(3.3,17.4)$ & $9(6.1,12.7)$ & 0.18 \\
Cumulative fluid overload \% at 48 h; median (IQR) & $6.6(3.1,10.3)$ & 0.04 \\
\hline
\end{tabular}




\section{What is AlReady Known?}

- Fluid overload leads to respiratory morbidity in children with acute lung injury.

What This Study Adds?

- Fluid overload in mechanically ventilated children is associated with increased organ dysfunction, longer duration of ventilation and ICU stay.

- Peak cumulative fluid overload occurs within first 5 days of admission requiring early identification.

overload at 48 hours was also associated with longer duration of mechanical ventilation $(P=0.001)$.

The duration of PICU stay was significantly higher among children with $\geq 15 \%$ cumulative maximum fluid overload [median (IQR): $13.5(7.75,22)$ days] as compared to that in children with $<15 \%$ cumulative maximum fluid overload [median (IQR): 6 (4, 10.75) days] on univariate as well as multivariate analysis ( $P<0.0001$ and $P<0.0001$, respectively) (Web Table II). Cumulative fluid overload at 48 hours was also significantly associated with longer duration of PICU stay $(P<0.0001)$.

\section{Discussion}

In this prospective observational study, we observed that there was no significant association of fluid overload with mortality or peak oxygenation index. However, the total duration of mechanical ventilation and duration of PICU stay was significantly higher in those with fluid overload $\geq 15 \%$. These children also had significant organ dysfunction as calculated using PELOD score.

We could not perform daily weight-based assessment of positive balance, which is considered to be a more objective method and better predictor of fluid balance; the severity of illness prevented daily weight measurements. In addition, the additional effect of the type of fluid used in such children was not measured though isotonic saline is used in management of children in our PICU uniformly. The other limitation was the sample size. On post hoc analysis to evaluate the association between cumulative maximum fluid overload percentage and mortality after adjusting for other independent factors, the power was estimated to be less than $40 \%$. Assuming similar cumulative maximum fluid overload and its effect on mortality with $80 \%$ power, the sample size required would be 862 . While we used diuretics for children with overt clinical features of fluid overload, we did not use renal replacement therapy solely for this purpose.

Unlike previous studies in children, we carried out a prospective study with a longer duration of follow up for outcomes. Also majority of previous studies were conducted in a restricted group of patients with acute lung injury $[6,10]$ acute kidney injury $[11,12]$ children on CRRT and hemodialysis [13-15]. We were able to study children commonly seen in a medical PICU with varied conditions to demonstrate the actual incidence and severity of fluid overload. We did not observe a relation between fluid overload and mortality which is similar to what has been reported previously $[16,17]$. However, few studies have shown association of fluid overload with mortality $[18,19]$. Previous studies have demonstrated significant effect of fluid overload on lung function and weaning and thereby the oxygenation index, duration of mechanical ventilation and ICU stay in both children and adults. Studies by Arikan, et al. [17] and Sinitsky, et al. [16] showed that peak fluid overload percent and fluid overload at 48 hours, respectively were associated with higher peak oxygenation index. Although we did not observe similar association of peak cumulative fluid overload percent of $\geq 15 \%$ with OI, the duration of ventilation as well as the ICU stay was significantly longer in this group. These findings are also similar to previous studies $[18,19]$.

Higher PELOD scores were observed in those with cumulative peak fluid overload of $\geq 15 \%$. However, it did not translate to a difference in mortality between the groups as other factors like increased severity of illness at presentation in both groups might have ultimately influenced the outcomes.

Possible mechanisms for an influence of fluid overload on morbidity are still not clear, though many studies claim it may be related to multiple organ dysfunction caused by increased interstitial edema and hypoperfusion especially in organs like lung and kidney [20,21]. Increased interstitial edema in the lungs leads to increased requirement of PEEP which would have adverse effects on the cardiac output and thereby the other organ systems [20,22,23]. In addition, excessive fluid resuscitation in critically-ill children contributes to 
the pathophysiology. Excessive 'leakiness' in such children which manifests as tissue edema, nonhydrostatic pulmonary edema (ARDS), and microalbuminuria are other contributing factors [24].

We conclude that fluid overload seems to be associated with significant morbidity, which includes increased organ dysfunction, longer duration of ventilation and ICU stay, in critically-ill mechanically ventilated children. Therefore, we recommend meticulous fluid management with daily and cumulative fluid balances which would help in early recognition of fluid overload in an intensive care setting. Larger adequately powered studies are needed to further address association of fluid overload with mortality.

Contributors: RL, SKK, JS: were involved in study design, analysis and writing of manuscript; SS: collected the data and wrote the manuscript. All authors read and approved the final manuscript.

Funding: None; Competing interest: None stated.

\section{REFERENCES}

1. Duke T, Molyneux EM. Intravenous fluids for seriously ill children: Time to reconsider. Lancet. 2003;362:1320-3.

2. Schrier RW, Berl T, Anderson RJ. Osmotic and nonosmotic control of vasopressin release. Am J Physiol. 1979;236:F32132.

3. Schrier RW, Goldberg JP. The physiology of vasopressin release and the pathogenesis of impaired water excretion in adrenal, thyroid, and edematous disorders. Yale J Biol Med. 1980;53:525-41.

4. Briassoulis G, Venkataraman S, Thompson AE. Energy expenditure in critically ill children. Crit Care Med. 2000;28:1166-72.

5. Wiedemann HP. A perspective on the fluids and catheters treatment trial (FACTT). Fluid restriction is superior in acute lung injury and ARDS. Cleve Clin J Med. 2008;75:42-8.

6. Valentine SL, Sapru A, Higgerson RA, Spinella PC, Flori HR, Graham DA, et al. Fluid balance in critically ill children with acute lung injury. Crit Care Med. 2012;40:2883-9.

7. Leteurtre S, Duhamel A, Grandbastien B, Lacroix J, Leclerc F. Paediatric logistic organ dysfunction (PELOD) score. Lancet. 2006;367:897.

8. Holliday MA, Segar WE. The maintenance need for water in parenteral fluid therapy. Pediatrics. 1957;19:823-32.

9. Slater A, Shann F, Pearson G, Paediatric index of mortality (PIM) study group. PIM2: A revised version of the paediatric index of mortality. Intensive Care Med. 2003;29:278-85.

10. Flori HR, Church G, Liu KD, Gildengorin G, Matthay MA. Positive fluid balance is associated with higher mortality and prolonged mechanical ventilation in pediatric patients with acute lung injury. Crit Care Res Pract. 2011;2011:854142.

11. Michael M, Kuehnle I, Goldstein SL. Fluid overload and acute renal failure in pediatric stem cell transplant patients. Pediatr Nephrol. 2003;19:91-5.

12. Kennedy CE, Akcan Arikan A. Fluid overload and kidney injury score (FOKIS). Pediatr Crit Care Med. 2014;15:132.

13. Gillespie RS, Seidel K, Symons JM. Effect of fluid overload and dose of replacement fluid on survival in hemofiltration. Pediatr Nephrol Berl Ger. 2004;19:1394-9.

14. Sutherland SM, Zappitelli M, Alexander SR, Chua AN, Brophy PD, Bunchman TE, et al. Fluid overload and mortality in children receiving continuous renal replacement therapy: The prospective pediatric continuous renal replacement therapy registry. Am J Kidney Dis. 2010;55:316-25.

15. Foland JA, Fortenberry JD, Warshaw BL, Pettignano R, Merritt RK, Heard ML, et al. Fluid overload before continuous hemofiltration and survival in critically ill children: A retrospective analysis: Crit Care Med. 2004;32:1771-6.

16. Sinitsky L, Walls D, Nadel S, Inwald DP. Fluid overload at 48 hours is associated with respiratory morbidity but not mortality in a general PICU: Retrospective cohort study. Pediatr Crit Care Med. 2015;16:205-9.

17. Arikan AA, Zappitelli M, Goldstein SL, Naipaul A, Jefferson LS, Loftis LL. Fluid overload is associated with impaired oxygenation and morbidity in critically ill children. Pediatr Crit Care Med. 2012;13:253-8.

18. Maitland K, Kiguli S, Opoka RO, Engoru C, OlupotOlupot $\mathrm{P}$, Akech SO, et al. Mortality after fluid bolus in African children with severe infection. N Engl J Med. 2011;364:2483-95.

19. Benakatti G, Singhi S, Jayshree M, Bansal A. Conventional vs. restrictive maintenence fluid regimen in children with septic shock after initial resusitation. Pediatr Crit Care Med. 2014;15:30.

20. Sakka SG, Klein M, Reinhart K, Meier-Hellmann A. Prognostic value of extravascular lung water in critically ill patients. Chest. 2002;122:2080-6.

21. Malbrain MLNG, Marik PE, Witters I, Cordemans C, Kirkpatrick AW, Roberts DJ, et al. Fluid overload, deresuscitation, and outcomes in critically ill or injured patients: A systematic review with suggestions for clinical practice. Anestezjol Intensywna Ter. 2014;46:361-80.

22. Schuller D, Mitchell JP, Calandrino FS, Schuster DP. Fluid balance during pulmonary edema. Is fluid gain a marker or a cause of poor outcome? Chest. 1991;100:1068-75.

23. Kuzkov VV, Kirov MY, Sovershaev MA, Kuklin VN, Suborov EV, Waerhaug K, et al. Extravascular lung water determined with single transpulmonary thermodilution correlates with the severity of sepsis-induced acute lung injury. Crit Care Med. 2006;34:1647-53.

24. Morris C, Plumb J. Mobilising oedema in the oedematous critically ill patient with ARDS: Do we seek natriuresis not diuresis? J Intensive Care Soc. 2011;12:92-7. 